\title{
NEVANLINNA CONSTANT AND ITS ANALOGUES FOR ENTIRE AND MEROMORPHIC FUNCTIONS OF FINITE NONINTEGRAL ORDER
}

\author{
TIEN-YU PETER CHERN ${ }^{(*)}$ AND S. M. SARANGI
}

\begin{abstract}
In this paper we deal with the Nevanlinna constant and its analogues for functions of finite positive nonintegral order.
\end{abstract}

\section{Introduction}

One of the central problems in the classical function theory is: for any complex value $\alpha$ and a given entire function $f(z)$ can we find connections between the growth of $f(z)$ and the value distribution of $\alpha$-points of $f(z)$. Borel (1897), Nevanlinna (1929), Shah (1944) Edrei and Fuchs (1960) among many others have attacked this kind of problems. The study on Nevanlinna constant has a long history and its results reflect partial achievements in this aspect.

In this paper we deal with the Nevanlinna constant and its analogues for functions of finite nonintegral order. In section 1 we begin with a Borel's inequality which expresses a relationship between the growth of a canonical product $P(z)$ and the value distribution of the zero-points of $P(z)$. The result (1.3) in Theorem 1 can be viewed as a version for entire functions connecting a result of the second author [7] (see also Theorem $G$ in this paper), both results (1.3) and (1.4) of Theorem 1 are new. The result (1.6) in Corollary is a quantitative version of a result of Valiron (see expression (1.2)) which has been proved by S. M. Shah in 1967 (see [13, Theorem 2]) by using a sequence of Pólya peaks; however our proof is simple and based on a result of the first author [2].

There are two kinds of variational results on the Nevanlinna constant in section 4. One kind of results considers $I(r, f)$, a mean of $T(r, f)$ instead of $T(r, f)$. The another kind of results deals with taking small meromorphic functions instead of taking complex values. The proof of Theorem 1 is left in section 5 .

Received October 20, 1998; revised November 25, 1998.

1991 Mathematics Subject Classification. Primary 30D20, 30D35.

Key words and phrases. Nevanlinna constant, nonintegral order.

" This paper of the first author was supported in part by the NSC R.O.C. 82-0208-M214-004. 


\section{Nevanlinna Constant for Entire Functions}

For a canonical product $P(z)$ of finite positive genus $p, 1897$ Borel has proved the following result:

Theorem A. (Borel's inequality)

$$
\log M(r, P)<K \int_{0}^{\infty}\left(n(t, P=0) r^{p+1} / t^{p+1}(t+r)\right) d t
$$

for a positive constant $K$. This Borel's inequality gives an indirectly relationship between $M(r, P)$ the maximum modulus function of $P$ and $n(r, P=0)$ the number of roots of the equation $P(z)=0$, in $|z| \leq r$.

Using above Borel's ineuality and the notion of the proximate order (for definition we refer the readers to [10]) for a given entire function $f$ of finite nonintegral order, 1913 Valiron [15] established a relationship between the growth of $f(z)$ and the value distribution of the zero-points of $f(z)$ as follows:

Theorem B. (Valiron)

$$
\limsup _{r \rightarrow+\infty} n(r, f=0) / \log M(r, f)>0
$$

In this paper we shall prove the following result and its analogues:

Theorem 1 . Let $f(z)$ be entire with finite nonintegral order $\lambda$. Let $\lambda(r)$ be a proximate order of $\log M(r, f)$ and $U(r, f)=r^{\lambda(r)}$ be a type function of $\log M(r, f)$. Then for each $a \in \mathbb{C}$, we have

$$
K_{U}(a, f) \geq(q+1-\lambda)(\lambda-q) / \lambda c_{1}(q)
$$

and

$$
\limsup _{r \rightarrow+\infty} \frac{n(r, f=a)}{U(r, f)} \geq(q+1-\lambda)(\lambda-q) / c_{1}(q)
$$

where $K_{U}(a, f)=\lim \sup _{r \rightarrow+\infty} \frac{N(r, f=a)}{U(r, f)} ; c_{1}(q)=1$, if $q=0, c_{1}(q)=2(q+1)[2+\log (q+$ 1)] if $q>0, q=[\lambda]$.

Remark. The result (1.3) in Theorem 1 can be viewed as an entire version of a result of the second author [7] (see also the below Theorem $G$ of this paper), both results (1.3) and (1.4) of Theorem 1 are new.

It follows from Theorem 1, we deduce immediately the following.

Corollary. Let $f(z)$ be entire with finite nonintegral order $\lambda$. Then for each $a \in \mathbb{C}$, we have

$$
\limsup _{r \rightarrow+\infty} \frac{N(r, f=a)}{\log M(r, f)} \geq(q+1-\lambda)(\lambda-q) / \lambda c_{1}(q) .
$$

and

$$
\limsup _{r \rightarrow+\infty} \frac{n(r, f=a)}{\log M(r, f)} \geq(q+1-\lambda)(\lambda-q) / c_{1}(q)
$$


Remark. In the above Corollary, the result (1.5) seems new and the expression (1.6) is a quantitative version of a result of Valiron (see expression (1.2)) which has been proved by S. M. Shah in 1967 (see [13, Theorem 2]). Shah proved it by making use a sequence of Pólya peaks.

Contrast to the result (1.6), there is a well known result as follows.

Theorem C. [1, p.55-59] [14, p.3] If $f$ is entire with order $\lambda, 0<\lambda<1$, and if all the zeros of $f(z)$ lie on the negative real axis and the counting function $n(r, f=$ $0) \sim K r^{\lambda}(K>0,0<\lambda<1)$ then

$$
\lim _{r \rightarrow+\infty} \frac{n(r, f=0)}{\log M(r, f)}=\frac{\sin \pi \lambda}{\pi},
$$

and

$$
\lim _{r \rightarrow+\infty} \frac{n(r, f=0)}{N(r, f=0)}=\lambda
$$

\section{Nevanlinna Constant for Meromorphic Functions}

We now state a result of Nevanlinna on Nevanlinna constant.

Applying Borel's inequality (1.1) again, in 1929 R. Nevanlinna [5, p.51] proved:

Theorem $\mathrm{D}$. (Nevanlinna) If $f$ is meromophic in the complex plane $\mathbb{C}$ with finite nonintegral order $\lambda$, then

$$
\limsup _{r \rightarrow+\infty} \frac{N(r, f=a)+N(r, f=b)}{T(r, f)} \geq \chi(\lambda)
$$

for distinct $a, b$ in $\overline{\mathbb{C}}$. This $\chi(\lambda)$ is called a Nevanlinna constant of $f$.

We next list some works after $\mathrm{R}$. Nevanlinna as following: For $0<\lambda<1$, 1944: Shah [9] proved:

Theorem $\mathbb{E}$. (Shah)

$$
\chi(\lambda) \geq 1-\lambda .
$$

For $0<\lambda<1$, in 1960 Edrei and Fuchs [3, p.236, Theorem 2] obtained the optimal value of $\chi(\lambda)$ by proving the following:

Theorem $\mathbb{F}$. (Edrei and Fuchs)

$$
\begin{aligned}
\chi(\lambda) & =1 \quad 0 \leq \lambda \leq 1 / 2 \\
& =\sin \pi \lambda \quad 1 / 2 \leq \lambda<1 .
\end{aligned}
$$

(see also [4, p.116, Theorem 4.14]).

The authors remark that in case $\lambda>1$, the optimal value of $\chi(\lambda)$ is not found yet.

If $f$ is meromorphic with finite nonintegral order $\lambda, 1978$ the second author [7] put

$$
K_{\lambda(r)}=: \limsup _{r \rightarrow+\infty} \frac{N(r, f=0)+N(r, f=\infty)}{U(r, f)}
$$


where $U(r, f)=r^{\lambda(r)}, \lambda(r)$ is a proximate order function of $T(r, f)$; and proved the following.

Theorem G. (Sarangi)

$$
K_{\lambda(r)} \geq(q+1-\lambda)(\lambda-q) / \lambda c_{1}(q)
$$

where $c_{1}(q)=1$, if $q=0, c_{1}(q)=2(q+1)[2+\log (q+1)]$ if $q>0, q=[\lambda]$.

1989 the second author and Patil [8] improved above Edrei and Fuchs' result (2.3) by proving

Theorem H. (Sarangi and Patil)

$$
\begin{aligned}
& K_{\lambda(r)} \geq 1 \quad \text { for } 0 \leq \lambda<1 / 2 \\
& K_{\lambda(r)} \geq \sin \pi \lambda \text { for } 1 / 2 \leq \lambda<1 .
\end{aligned}
$$

We remark that $K_{\lambda(r)}$ is an analogy of $\chi(\lambda)$ and is subtler than $\chi(\lambda)$.

\section{A Lemma}

To prove Theorem 1, we need a result of the first author as follows.

Lemma 1. [2, lemma 4.1] Let $f$ be nonconstant meromorphic in $\mathbb{C}$, and $S(r)$ be an unbounded increasing function of finite positive order $\lambda$. If $a \in \overline{\mathbb{C}}=\mathbb{C} \cup\{\infty\}$, then we have

$$
\limsup _{r \rightarrow+\infty} \frac{N(r, f=a)}{U(r)} \leq(1 / \lambda) \limsup _{r \rightarrow+\infty} \frac{n(r, f=a)}{U(r)}
$$

where $U(r)=r^{\lambda(r)}$ is a type function of $S(r)$ and $\lambda(r)$ is a proximate order function of $S(r)$.

The above Lemma 1 and the meaning of $K_{\lambda(r)}$ gives:

Proposition 1 . If $f$ is meromorphic with finite nonintegral order $\lambda$,. then

$$
\limsup _{r \rightarrow+\infty} \frac{n(r, f=0)+n(r, f=\infty)}{T(r, f)} \geq \limsup _{r \rightarrow+\infty} \frac{n(r, f=0)+n(r, f=\infty)}{U(r, f)} \geq \lambda K_{\lambda(r)} .
$$

The results of Proposition 1 can be viewed as analogues of Theorem $D$ in terms of $n(r, a)$ instead of in terms of $N(r, a)$.

\section{More Variational Results}

For any nonconstant meromorphic function $f(z)$, in 1929 R. Nevanlinna [5, p.25] introduced a mean value of $T(r, f)$ :

$$
I(r, f)=: \frac{1}{r} \int_{0}^{r} \log ^{+} M(t, f) d t
$$


and prove that:

Thereom H. (Nevanlinna) For $k>1$, we have

$$
I(r, f) \leq \frac{k+1}{k-1} T(k r, f) .
$$

If $f(z)$ is of finite positive order $\lambda$, then $I(r, f)$ is dominated above by $U(r, f)$ which is given by a well known classical result:

Theorem I. If $0<\lambda<+\infty$, then

$$
I(r, f) \leq d(\lambda) U(r, f)
$$

where $d(\lambda)=\frac{(1-\lambda)+\left(1+\lambda^{2}\right)^{1 / 2}}{(1+\lambda)+\left(1+\lambda^{2}\right)^{1 / 2}}\left(\frac{\lambda}{1+\left(1+\lambda^{2}\right)^{1 / 2}}\right)^{\lambda}$, and $U(r, f)$ is a type function of $T(r, f)$.

In terms of $I(r, f)$, there are two well known results.

Theorem J. ([6, Theorem 2], [11, Theorem 2]) If $f$ is meromorphic with finite nonintegral order $\lambda$, then

$$
\limsup _{r \rightarrow+\infty} \frac{N(r, f=0)+N(r, f=\infty)}{I(r, f)} \geq 0,
$$

and

$$
\limsup _{r \rightarrow+\infty} \frac{n(r, f=0)+n(r, f=\infty)}{I(r, f)} \geq 0 .
$$

The result (4.4) is due to Okada which can be viewed as an alternating version of Theorem D. The result (4.5) is due to Shah which can be viewed as a meromorphic version of (1.2) a result of $G$. Valiron. In this aspect we have the following:

Theorem 2. If $f$ is meromorphic with finite nonintegral order $\lambda$, then

$$
\limsup _{r \rightarrow+\infty} \frac{N(r, f=0)+N(r, f=\infty)}{I(r, f)}>\frac{K_{\lambda(r)}}{d(\lambda)}
$$

and

$$
\limsup _{r \rightarrow+\infty} \frac{n(r, f=0)+n(r, f=\infty)}{I(r, f)}>\frac{\lambda K_{\lambda(r)}}{d(\lambda)}
$$

Remarks. In Theorem 2, (4.6) improves the right hand of (4.4) by replacing the lower bound 0 by a positive number $K_{\lambda(\lambda)} / d(\lambda)$ and (4.7) improves the right hand of (4.5) by replacing the lower bound 0 by a positive number $\lambda K_{\lambda(r)} / d(\lambda)$. On the proofs of Okada and Shah, they have used a gerneralized version of the Borel's inequality of (1.1) and the existence of a sequence of Pólya peak for functions of finite positive order. Our proof is based on a key inequality which is due to Chern and is stated in the Lemma 1.

The proof of Theorem 2. (4.3) and (2.4) gives (4.6). (4.6) and Lemma 1 implies (4.7). 
Let's turn to the results taking small functions instead of assuming complex values by the given function. Let $g_{1}(z)$ and $g_{2}(z)$ be two distinct meromorphic funciton (including rational functions or finite constants) which are small with respect to $f(z)$ in the sense that

$$
T\left(r, g_{i}(z)\right)=o(T(r, f)), \quad(i=1,2) \quad \text { as } r \rightarrow+\infty .
$$

Theorem 3. Let $f$ be meromorphic with finite nonintegral order $\lambda$. If $g_{i}(z)(i=1,2)$ are two distinct meromorphic functions which are small with respect to $f(z)$ and $k>0$, then

$$
\limsup _{r \rightarrow+\infty} \frac{N\left(r, f=g_{1}\right)+N\left(r, f=g_{2}\right)}{T(k r, f)} \geq(q+1-\lambda)(\lambda-q) / \lambda c_{1}(q) k^{\lambda},
$$

and

$$
\limsup _{r \rightarrow+\infty} \frac{n\left(r, f=g_{1}\right)+n\left(r, f=g_{2}\right)}{T(k r, f)} \geq(q+1-\lambda)(\lambda-q) / c_{1}(q) k^{\lambda},
$$

Theroem 3 is an analogue of a result of Shah [12, p.811, Theorem 2] and is an immediately consequence of the following.

Theorem 4. Let $f$ be meromorphic with finite nonintegral order $\lambda$. If $g_{1}(z)$ and $g_{2}(z)$ are two distinct meromorphic functions which are small with respect to $f(z)$ and $k>0$, then

$$
\limsup _{r \rightarrow+\infty} \frac{N\left(r, f=g_{1}\right)+N\left(r, f=g_{2}\right)}{U(k r, f)} \geq(q+1-\lambda)(\lambda-q) / \lambda c_{1}(q) k^{\lambda},
$$

and

$$
\limsup _{r \rightarrow+\infty} \frac{n\left(r, f=g_{1}\right)+n\left(r, f=g_{2}\right)}{U(k r, f)} \geq(q+1-\lambda)(\lambda-q) / c_{1}(q) k^{\lambda}
$$

where $U(r, f)$ is a type function of $T(r, f)$.

The proof of Theorem 4. If we put

$$
F(z)=\frac{f(z)-g_{1}(z)}{f(z)-g_{2}(z)}
$$

and denote the type function of $T(r, F)$ by $U(r, F)$.

Noticing that

$$
U(r, f) \sim U(r, F)
$$

and

$$
U(k r, f) \sim k^{\lambda} U(r, f),
$$

it follows from Theorem $G$, we have (4.11). (4.11) and Lemma 1 deduce (4.12). This completes the proof of Theorem 4 . 


\section{The Proof of Theorem $\mathbb{1}$}

For each $a \in \mathbb{C}$, put

$$
f(z)-a=z^{k} e^{Q(z)} P(z),
$$

where $P(z)$ is the canonical product forms of non-zero a-points of $f(z), Q(z)$ is a polynomial of degree $\leq q$. Hence we have

$$
\log M(r, f) \sim \log M(r, 1 /(f-a)) \sim \log M(r, P) .
$$

By Hayman [4, pp.27-29], we have

$$
\log M(r, P) \leq c_{1}(q)\left\{q r^{q} \int_{0}^{r} \frac{N(t)}{t^{q+1}} d t+(q+1) r^{q+1} \int_{r}^{\infty} \frac{N(t)}{t^{q+2}} d t\right\}
$$

where $N(t)=N(t, f=a)$.

Put

$$
K_{U}(a, f)=\limsup _{r \rightarrow+\infty} \frac{N(r, f=a)}{U(r, f)} .
$$

Since $K_{U}(a, f) \leq 1$, for each $\epsilon>0$, there is a $r_{0}$ such that

$$
N(r, a) \leq\left(K_{U}(a, f)+\epsilon\right) U(r, f) \text { for } r \geq r_{0}
$$

hence for sufficiently large $r$, we have

$$
\begin{aligned}
& \log M(r, f) \leq c_{1}(q)\left\{q r^{q} \int_{0}^{r} \frac{\left(K_{U}(a, f)+\epsilon\right) t^{\lambda(t)}}{t^{q+1}} d t\right. \\
& \left.+(q+1) r^{q+1} \int_{r}^{\infty} \frac{\left(K_{U}(a, f)+\epsilon\right) t^{\lambda(t)}}{t^{q+2}} d t\right\}+O\left(r^{q}\right) \\
& =c_{1}(q)\left(K_{U}(a, f)+\epsilon\right)\left\{q r^{q} \int_{0}^{r} t^{\lambda(t)-(q+1)} d t\right. \\
& \left.+(q+1) r^{q+1} \int_{r}^{\infty} t^{\lambda(t)-(q+2)} d t\right\}+O\left(r^{q}\right) .
\end{aligned}
$$

Since

$$
\int_{0}^{r} t^{\lambda(t)-(q+1)} d t \sim \frac{r^{\lambda(r)-q}}{\lambda-q}
$$

and

$$
\int_{r}^{\infty} t^{(\lambda(t)-(q+2))} d t=\frac{r^{(q+1-\lambda(r))}}{q+1-\lambda}
$$

we have

$$
1=\limsup _{r \rightarrow+\infty} \frac{\log M(r, f)}{U(r, f)} \leq c_{1}(q)\left(K_{U}(a, f)+\epsilon\right)\left\{\frac{1}{(\lambda-q)}+\frac{1}{(q+1-\lambda)}\right\} .
$$


Therefore we deduce (1.3). (1.3) and Lemma 1 gives (1.4). This completes the proof of Theorem 1.

\section{Acknowledgement}

The authors are grateful for the referee's helpful suggestions on this paper.

\section{References}

[1] R. P. Jr. Boas, Entire Functions, Academic Press, New York, 1954.

[2] T. Y. Peter Chern, A defect relation and its variations for meromorphic functions, (preprint).

[3] A. Edrei and W. H. J. Fuchs, The deficiencies of meromorphic functions of order less than one, Duke Math. J. 27(1960), 233-249.

[4] W. Hayman, On Meromorphic Functions, Clarendon Press, Oxford, 1964.

[5] R. Nevanlinna, Le Théorème de Picard-Borel et la Théorie des Fonctions, Méromorphes, Coll. Borel, 1929.

[6] Y. Okada, Some theorems on meromorphic functions, Proc. Amer. Math. Soc. $1(1950)$, 246-249.

[7] S. M. Sarangi, On $\rho(r)$-defect and Picard Borel Theorem, Mat. Science Publications, Madras (1978), 182-189.

[8] S. M. Sarangi and S. J. Patil, On deficient values of meromorphic functions, Journal of Math. Research and Exposition 9(1989), 25-30.

[9] S. M. Shah, A note on meromorphic functions, The Math. Student (1944), 67-70.

[10] S. M. Shah, On proximate orders of integral functions, Bull. Amer. Math. Soc. 52(1946), 326-328.

[11] S. M. Shah, Some theorems on meromorphic functions, Proc. Amer. Math. Soc. 2(1951), 694-698.

[12] S. M. Shah, Meromorphic functions of finite order, Proc. Amer. Math. Soc. 10(1959), 810-821.

[13] S. M. Shah, Entire funcitons with no finite deficient value, Arch. Rational Mech. Anal. 26(1967), 179-187.

[14] S. K. Singh and H. S. Gopalakrishna, Grouth of entire and meromorphic functions, Comment. Math. Univ St. Pauli, 26(1977), 3-15.

[15] G. Valiron, Lecture on General Theory of Integral Functions, Toulouse, 1923, (reprinted), Chelsea, 1949.

Department of Applied Mathematics, I-Shou university, Ta Hsu, Kaohsiung County, 84008 Taiwan.

Department of Studies in Mathematics, Karnatak University, Pavate Nagar, Dharward-580 003, Karnataka-India. 